\title{
Modelado estadístico de pronóstico de esfuerzo de construcción de Software
}

\section{Statistical modeling of software construction effort forecasting}

\author{
LÓPEZ, Gilberto†** \& SORIA, Myriam \\ Universidad Tecnológica de León \\ ID $1^{\text {er }}$ Autor: Gilberto, López / ORC ID: 0000-0002-9431-1656, Researcher ID Thomson: S-4615-2018, CVU CONACYT \\ ID: 946903 \\ ID $1^{\text {er }}$ Coautor: Myriam, Soria / ORC ID: 0000-0001-5718-9949, Researcher ID Thomson: V-9022-2019, CVU \\ CONACYT ID: 772647
}

DOI: $10.35429 /$ JCA.2019.9.3.6.10

Recibido Enero 10, 2019; Aceptado Marzo 30, 2019

\section{Resumen}

Este trabajo se centra en la estimación del esfuerzo de construcción de software basado en el rendimiento del programador. Esta estimación es de gran importancia para la organización, ya que se requieren mejoras de los procesos que utilicen buenas prácticas, como lo es el CMMI (Capability Maturity Model Integration) Por lo cual, se realizó una investigación descriptiva para caracterizar el desempeño en la construcción de software. Los productos de software son requisitos que responden a las necesidades específicas de un cliente y sus dependencias técnicas no requeridas explícitamente por el cliente, pero necesarias para el cumplimiento de un requisito explícito. Los desempeños caracterizados permiten a una organización saber si sus procesos están bajo control, así como producir modelos para estimar esfuerzos y costos que sean confiables.

Estimación. Esfuerzo. Software

\begin{abstract}
This work focuses on the estimation of software construction effort based on programmer performance. This estimate is of great importance for the organization, since improvements are required of processes that use good practices, such as CMMI (Capability Maturity Model Integration). Therefore, a descriptive investigation was carried out for characterize the construction performance of software. Software products are requirements that respond to the specific needs of a customer and their technical dependencies not explicitly required by the customer, but necessary for the fulfillment of an explicit requirement. Characterized performances allow an organization to know if its processes are under control, as well as to produce models to estimate efforts and costs that are reliable.
\end{abstract}

Software. Effort. Estimation

Citación: LÓPEZ, Gilberto \& SORIA, Myriam. Modelado estadístico de pronóstico de esfuerzo de construcción de Software. Revista de Cómputo Aplicado. 2019, 3-9: 6-10

\footnotetext{
* Correspondencia al Autor (Correo electrónico: gpadilla@utleon.edu.mx)
}

$\uparrow$ Investigador contribuyendo como primer Autor. 


\section{Introducción}

La UTL necesita producir estimaciones de esfuerzo que estén apegadas a modelos de calidad, sin embargo, no conoce el desempeño de sus procesos de construcción de software ni si estos están en control. Diversos modelos de calidad y autores comparten conceptos y los definen de manera similar, tal como el concepto de calidad en sí. Según Juran ${ }^{1}$ la "Calidad es que un producto sea adecuado para su uso" o la ASQ sostiene que la calidad es "un producto o servicio libre de deficiencias", y la ISO-9000:2005 indica que la calidad es "el grado en el que unos conjuntos de características inherentes cumplen con los requisitos". ${ }^{2}$

En términos de satisfacción, la calidad debe controlarse para entregar productos y servicios que cumplan con las expectativas del cliente; y metodologías como seis sigmas establecen niveles empíricos que tengan un balance entre los que se espera como calidad y el esfuerzo que se requiere para cumplir con ella, esto es: si se conocen los desempeños de los procesos y estos están caracterizados con media y desviación estándar, entonces los límites naturales de ese proceso incluyen el $99.97 \%$ de los eventos monitoreados. Por ejemplo, un producto está "libre" de defectos, si los defectos que pueden presentarse en un producto entregado no son más del promedio de defectos observados históricamente más 3 desviaciones estándar, o sea, se entregó un producto sin los defectos previsibles dentro de 3 desviaciones estándar por debajo y por encima de la media; es decir seis sigmas "sin defectos".

Establecido lo anterior, se pueden producir previsiones o pronósticos relevantes a la calidad poniendo como base los desempeños caracterizados, con media y desviación estándar, de los procesos y con estas previsiones establecer objetivos organizacionales de calidad.

\section{Hipótesis Nula:}

Los desempeños de construcción de software no se comportan de manera normal.

Hipótesis Alternativa:

Los desempeños de construcción de software se comportan de manera normal.

\author{
es $p>0.05$
}

El criterio para descartar la hipótesis nula

\section{Objetivo general:}

Caracterizar los desempeños de construcción de pantallas y código de la unidad de producción de software de la UTL.

\section{Objetivos particulares:}

$\begin{array}{ll}- & \text { Recolectar de datos para análisis } \\ - & \text { Analizar de desempeño Senior (Sn) } \\ \text { - } & \text { Analizar de desempeño Junior (Jn) } \\ \text { - } & \text { Integrar desempeños en el pronóstico de } \\ & \text { esfuerzo. }\end{array}$

\section{Metodología}

\section{Tipo de investigación}

Se realizó una investigación descriptiva para caracterizar desempeños en la construcción de software, centrándonos en el "qué" se debería medir entendiendo la naturaleza del producto $\mathrm{y}$ la forma en que se construye.

\section{Recolección de datos}

Parte esencial del proyecto fue la recolección de datos, para ello se mantuvo un monitoreo constante de las actividades de construcción de pantallas y código, este monitoreo se llevó a cabo con la cooperación de los desarrolladores, ellos usaron una herramienta llamada de manera genérica "play/stop"; con ella los desarrolladores registraron el inicio de sus actividades, las pausas y el fin de la actividad de construcción. Las actividades de construcción fueron determinadas por los requerimientos funcionales y sus derivados, estos requerimientos responden a necesidades específicas del cliente y los derivados son aquellas dependencias para cumplir con los requerimientos funcionales, de manera que cada requerimiento corresponde a una actividad en alguna fase de construcción.

\footnotetext{
1995

2 GUTIÉRREZ PULIDO, DE LA VARA SALAZAR, 2013 


\section{Análisis de datos}

Con el registro se elaboró una tabla en la que se relacionó cada actividad de construcción y el esfuerzo en horas requerido para completar la tarea y con esa relación se sometieron los datos a pruebas de normalidad, Anderson-Darling, usando la herramienta estadística Minitab. Los análisis estadísticos arrojaron para cada conjunto de datos métricas de media, desviación estándar $\mathrm{y}$ valor $\mathrm{p}$, siendo el valor $\mathrm{p}$ el criterio principal para determinar si el conjunto de datos seguía una distribución normal.

Con las métricas características de los procesos de se determinó que ellos estaban en control y, que las métricas eran confiables para producir modelos de estimación predictivos.

Las métricas obtenidas caracterizan desempeños Junior ( $\mathrm{Jr}$ ) y Senior ( $\mathrm{Sn}$ ) de los procesos de construcción de pantallas y código, que fueron utilizadas para alimentar un modelo de estimación usando el método Montecarlo.

\section{Resultados}

En cuanto a los desempeños se puede afirmar que se comportan de forma normal a nivel de requerimiento, de ello se presenta el siguiente resumen:

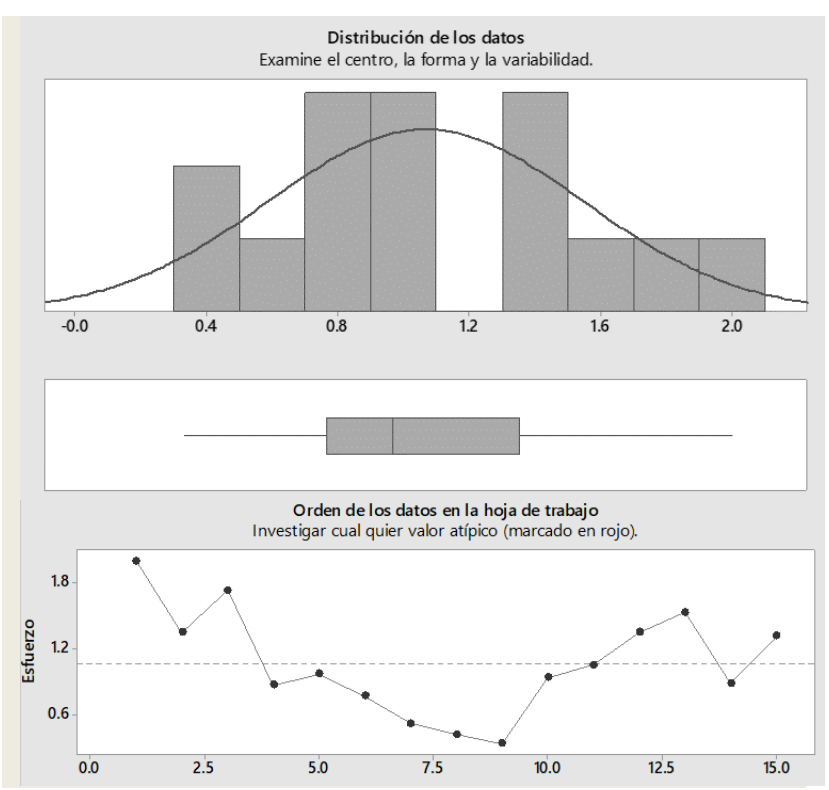

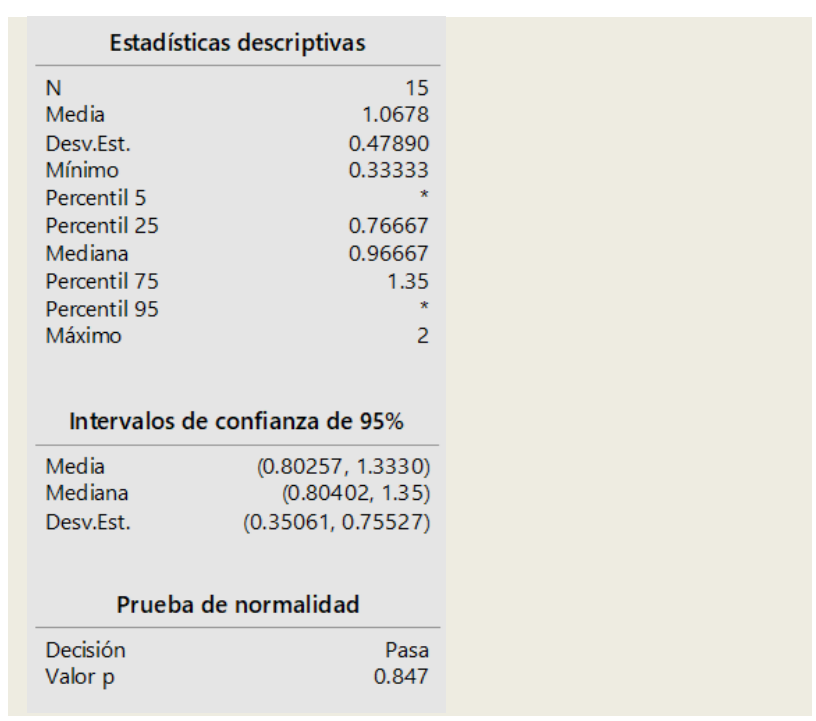

Gráfico 1 Caracterización de Desempeños Senior(SN) en pantallas

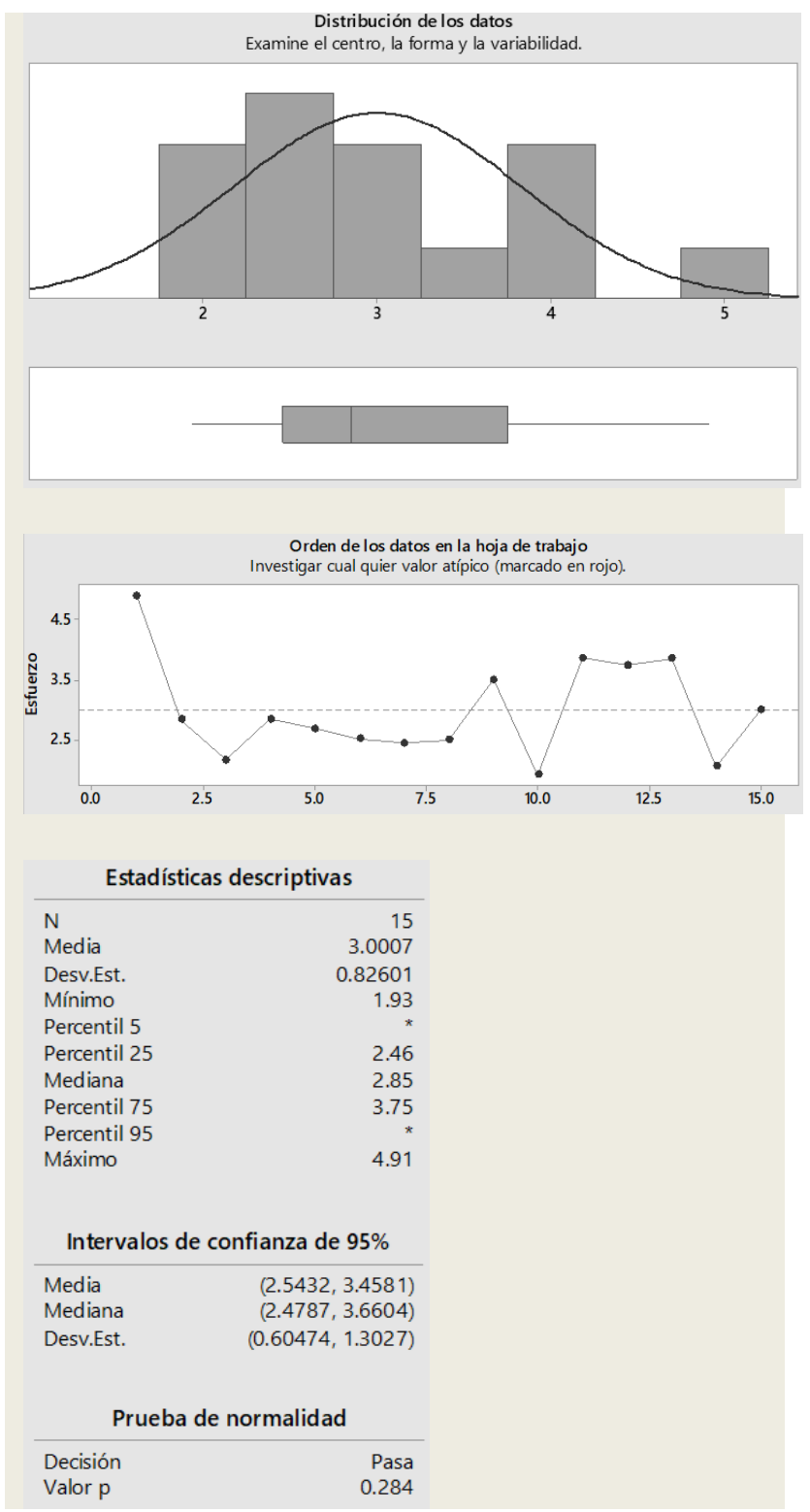

Gráfico 2 Caracterización de Desempeños Senior(SN) en codificación 


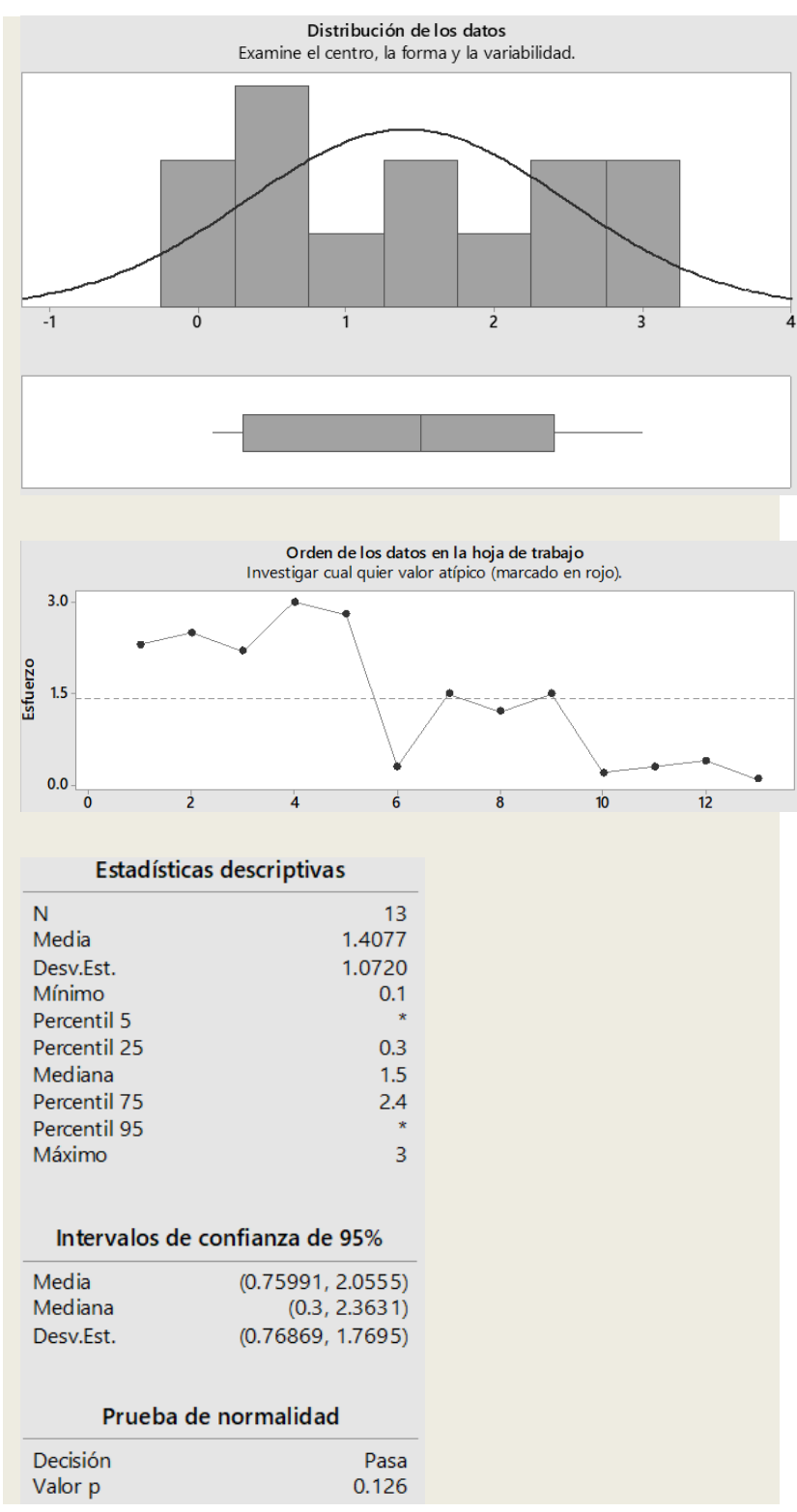

Gráfico 3 Caracterización de Desempeños Junior(JN) en pantallas

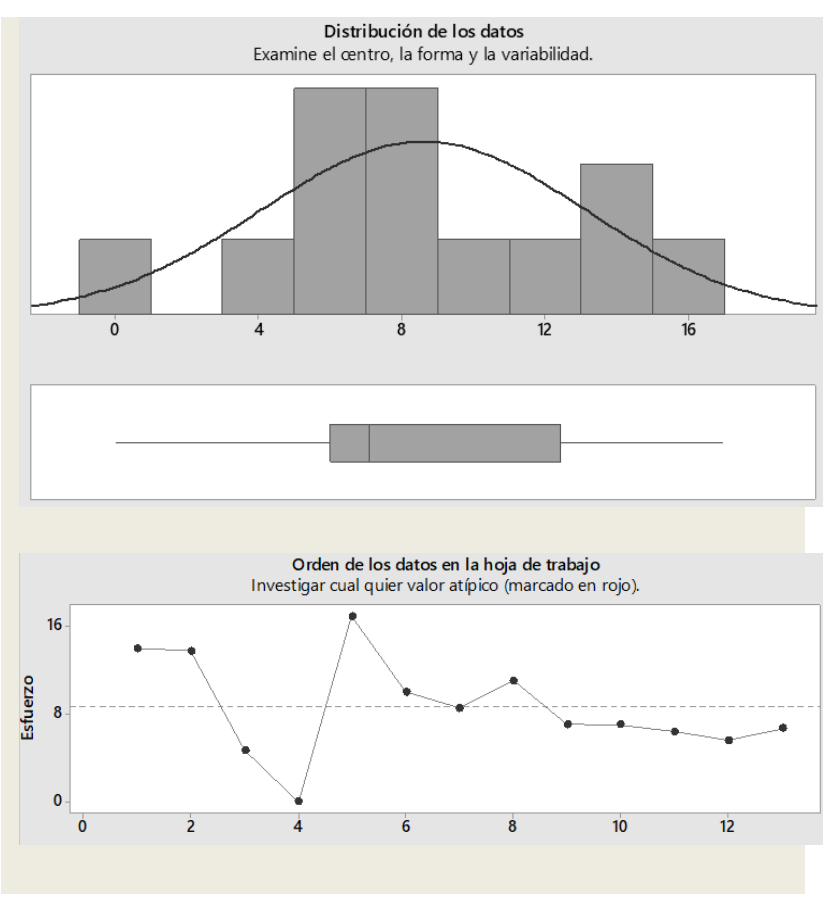

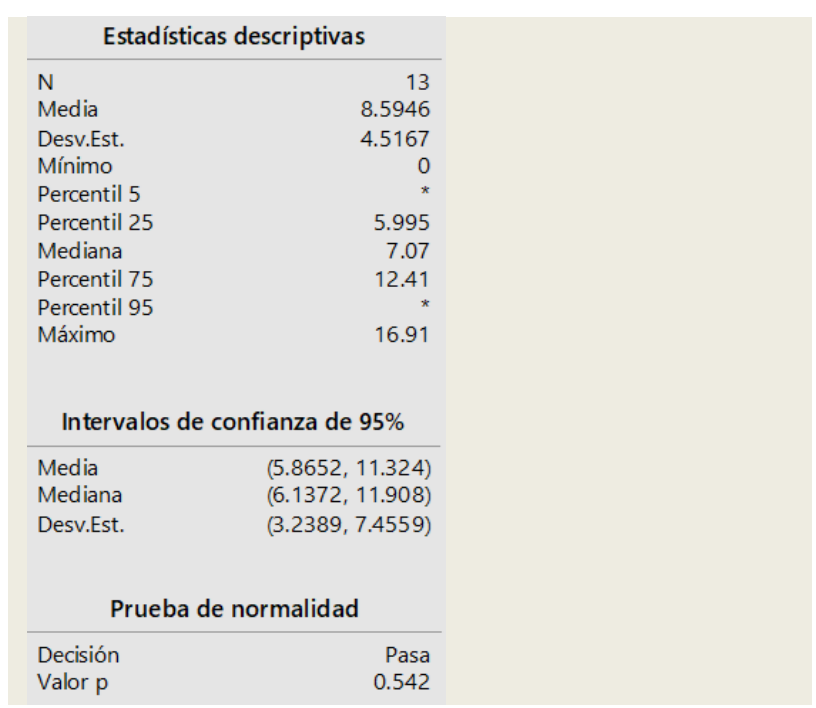

Gráfico 4 Caracterización de Desempeños Junior(JN) en codificación

Con los valores de media y desviación estándar de los desempeños el esfuerzo se logró estimar por actividad de construcción usando software de simulación Montecarlo, en particular, la extensión Crystal Ball de Oracle para MS Excel. El siguiente es un ejemplo de pronóstico de esfuerzo para un proyecto con un nivel de certeza del $80 \%$.

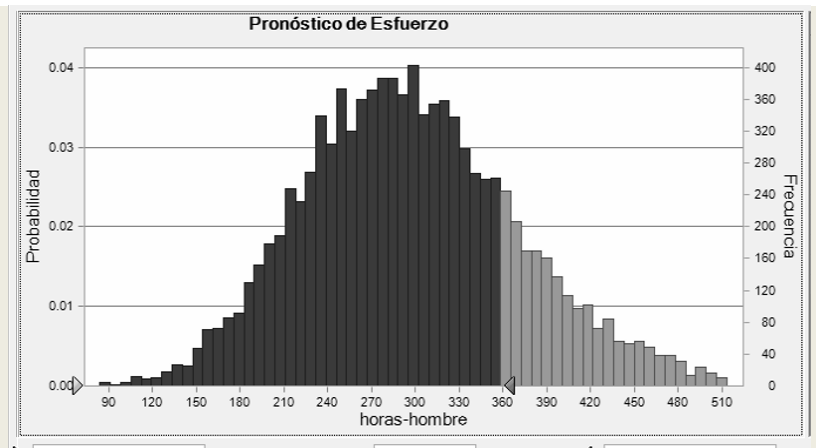

\begin{tabular}{|c|c|}
\hline Estadistica & Valores de previsión \\
\hline Pruebas & 10.000 \\
\hline Caso base & 298 \\
\hline Media & 299 \\
\hline Mediana & 293 \\
\hline Modo & 188 \\
\hline Desviación estándar & 77 \\
\hline Varianza & 5866 \\
\hline Sesgo & 0.4555 \\
\hline Curtosis & 3.50 \\
\hline Coeficiente de variaci & 0.2566 \\
\hline Mínimo & 51 \\
\hline Máximo & 710 \\
\hline Error estándar medio & 1 \\
\hline
\end{tabular}

Grafico 5 Pronóstico de esfuerzo para un proyecto con un nivel de certeza del $80 \%$

LÓPEZ, Gilberto \& SORIA, Myriam. Modelado estadístico de pronóstico de esfuerzo de construcción de Software. Revista de Cómputo Aplicado. 2019 


\section{Conclusiones}

El proceso de construcción de pantallas está en control pues no se encontraron puntos atípicos, tanto SN como JR.

El proceso de construcción de código está en control pues no se encontraron puntos atípicos, tanto SN como JR.

Los desempeños se comportan normales a nivel de requerimiento puesto que están clasificados de acuerdo con un estándar local basado en el método PROBE, sin embargo, al ser tan específico para nuestra organización no puede considerarse como una medición estadística internacional, necesaria para demostrar los objetivos del nivel 4 de CMMI.

En esta investigación no se tomó en cuenta el tamaño de la actividad: La complejidad del requerimiento fue muy similar y la normalidad de la distribución de los datos de los desempeños no se afectó; pero surge la duda de si la complejidad de los requerimientos difiere mucho de uno a otro, produciendo grandes diferencias entre los tamaños de las actividades de construcción, pueda producir una de falta de normalidad en la distribución de los datos.

El siguiente paso será analizar el desempeño como una tasa que esté dada por el tamaño del requerimiento con respecto al esfuerzo requerido para construirlo. $\mathrm{La}$ determinación del tamaño del requerimiento deberá realizarse con una medida estándar y reconocida internacionalmente, esto con la finalidad de poder aspirar los objetivos del nivel 4 de CMMI.

\section{Referencias}

CMMI Product Team. (2010). CMMI® for Development, Version 1.3. Pittsburgh, PA.: Carnegie Mellon University Press.

Gutiérrez Pulido, H., \& De la Vara Salazar, R. (2013). Control estadístico de la calidad y Seis Sigma. México, D.F.: Mc Graw Hill .

Juran, J. M., \& Gryna, F. (1995). Análisis y planeación de la calidad. México: McGrawHill. 